\title{
The development of a virtual camera system for astronaut-rover planetary exploration
}

\author{
Donald W. Platt ${ }^{\mathrm{a},}$ and Guy A. Boy ${ }^{\mathrm{a}}$ \\ ${ }^{a}$ Human-Centered Design Institute, Florida Institute of Technology, $150 \mathrm{~W}$. University Blvd, Melbourne, FL, \\ 32935, USA
}

\begin{abstract}
A virtual assistant is being developed for use by astronauts as they use rovers to explore the surface of other planets. This interactive database, called the Virtual Camera (VC), is an interactive database that allows the user to have better situational awareness for exploration. It can be used for training, data analysis and augmentation of actual surface exploration. This paper describes the development efforts and Human-Computer Interaction considerations for implementing a firstgeneration VC on a tablet mobile computer device. Scenarios for use will be presented. Evaluation and success criteria such as efficiency in terms of processing time and precision situational awareness, learnability, usability, and robustness will also be presented. Initial testing and the impact of HCI design considerations of manipulation and improvement in situational awareness using a prototype $\mathrm{VC}$ will be discussed.
\end{abstract}

Keywords: Situation Awareness, Augmented Reality, Human-Computer Interaction, Tablet Computing, Usability Testing.

\section{Introduction}

The Virtual Camera (VC) is an interactive database system designed to improve situational awareness for planetary exploration and operation of human-piloted rovers on the surface of other planetary bodies. It can be used for training, data analysis and augmentation of actual surface exploration [1].

With new emphasis on human asteroid exploration at NASA the VC is also being designed to be used in assistance for asteroid exploration using vehicles that can fly very close to and perhaps anchor on a nearEarth asteroid (NEA). In this environment the main issues will be providing situational awareness to allow close to asteroid navigation and near real-time sensor data updates. A learning mechanism would also be useful to allow the system to provide additional useful information to the user as the implementation progresses.

This paper will illustrate the basic tenants of human-centered design as it is applied to the development of the VC for space exploration.

\section{Virtual Camera Design Considerations}

\subsection{Design Summary}

The VC is a tool to assist in the exploration of other planetary bodies. There are a variety of data sources that will need to be integrated or fused to create the interactive VC database. These include previous terrain and elevation data for the body under exploration, lidar and radar data and surface composition data. The goal of the $\mathrm{VC}$ is to turn all of this data into strategic knowledge for exploration. There is a level of complexity in the data fusion process. How can multiple data sources be represented in a coherent user interface? How can the system represent and display knowledge derived from this data fusion taking into account expertise from those involved in space exploration such as astronauts, mission operations personnel and scientists.

\footnotetext{
${ }^{*}$ Corresponding author. E-mail: dplatt@fit.edu.
} 


\subsection{User Considerations}

An astronaut's workload will be very high as they explore another planet. As workload increases level of stress also increases. A point is reached where stress causes a discontinuity and performance "crashes." The VC must assist in workload by assisting in navigation, planning of safe traverses and in determining areas of interest to explore.

The VC must be able to recover from errors and continue to operate in a meaningful way. It will use feedback to determine future action. Figure 1 shows the feedback loop used by the VC for displaying data and information for users. It will then determine if the data was useful or not and be used in its learning mechanism. Feedback should be clear and verbose enough for the users to understand what the system is currently doing.

Errors against constraints/rules should be checked as much as possible. For instance, if a mission planner "draws" a planned traverse which will take the rover over dangerous terrain a message should appear explaining the danger and perhaps suggesting alternatives.

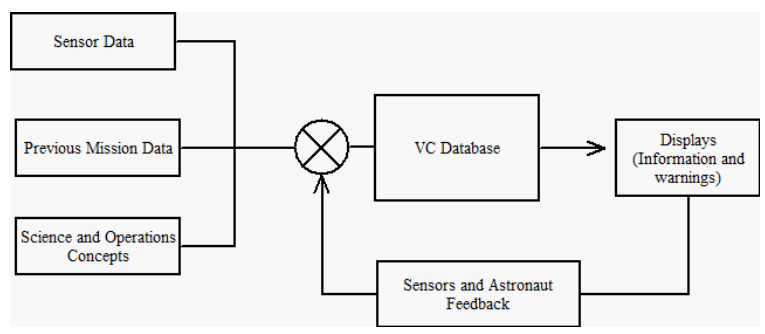

Figure 1. Feedback Loop for VC Data Display

Mission operations personnel will be interested in using the $\mathrm{VC}$ for mission planning purposes as well as training. For the actual mission the large distances involved between Earth and the body being explored means that communications delays will make it impossible to provide real-time feedback to astronauts. This requires the VC to capture the expertise of the mission operations personnel on-board. The goal is to make the $\mathrm{VC}$ a remote agent for the mission operations personnel.

Scientists will also use the VC for planning future traverses and to look for new discoveries on the body being explored. Their experience level with the VC tool will typically be less than the astronauts and mission operations personnel.
It is also anticipated the VC could be offered to the general public to explore and encourage learning and interested in Science, Technology, Engineering and Mathematics (STEM) disciplines. It is also possible they could be involved to explore and find safe traverses. For example, a game could be developed and distributed to find the most efficient routes for exploration that could actually be used by the astronauts on the real mission.

The general public will have limited knowledge but can be trained to recognize patterns and Areas of Interest. They will use the VC for entertainment, learning, exploration and to satisfy curiosity.

\subsection{Data Considerations}

The VC will begin by using data from historical missions to the body to be explored. This data will be augmented over time by future missions as data becomes available as well as data collected by the exploration vehicle the VC is connected to.

- Data collected from a variety of sensors:

- Imaging data (stereo cameras)

- Active and adaptive optics

- Spectrometers

- Laser/lidar instruments

Different data from different missions and different sensors will have different resolutions and data formats. This creates a great challenge for the $\mathrm{VC}$ system [2]. Adding one more data type or improving the resolution of available datasets the number of uses as well as the importance of the information conveyed will increase in a non-linear fashion.

Data needs to be projected in three dimensions for realism. This creates an abstraction model of the real body being explored. The format needs to be easy for human users to interact with and gain knowledge about. Human users can visualize in 3-D and recognize patterns. Oftentimes humans can see more useful information in patterns than just in series of number.

The VC will take advantage of these capabilities.

Three-dimensions of terrain information can be augmented with colors to add a fourth dimension of information. This will allow a natural representation of data in easily recognized terrain patterns. With the additional use of symbols and colors more dimensions can be added to the 3-D terrain model. The result is a realistic digital terrain model of the surface being explored.

Figure 2 is an example of a undersea mountain (seamount) with elevation data plotted in $\mathrm{x}$ and $\mathrm{y}$ coordinates. Another dimension is added by representa- 
tive colors. This additional dimension could be data such as mineral content, water content, or a safety concern such as loose terrain sensed by radar.

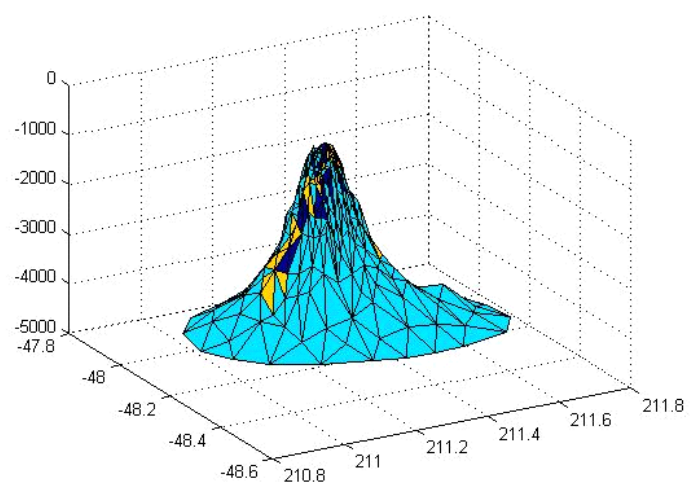

Figure 2 An Example of a 3-D Representation of Sea Mount Elevation Data Combined with a Fictitious Forth Data Dimension (yellow and blue colors) that could represent resources or safety concerns

It is also hoped that the VC can take advantage of tools already available for mission planning and science exploration such as the NASA Ames xGDS toolset [3]. The advantage of the VC architecture is the portability to take a subset of data such as in the xGDS system and translate it into a remote agent that can be used remotely by astronauts exploring the solar system.

\subsection{Categorizations}

Categorization is the process in which objects are recognized, differentiated, and understood. The VC will make use of categorization to assist in identifying areas of interest or of safety concern. For instance rocks that are flat may indicate sedimentation that could be indicative of a place where water flowed previously and perhaps may have fossil bacteria which may be of scientific interest. Algorithms are being developed to look for surface features autonomously in the Mars Exploration Rover missions [4].

Colors and symbols should be used to represent similar objects -rocks or geological formations of interest, areas of danger, areas requiring further exploration for instance (for example, a red exclamation point could mean danger).

Possible categories could include:

- Rock types

- Crater types

- Terrain slopes

- Bolder sizes
- Areas that may contain water or other resources

- Other geological features

\section{User Requirements Elicitation}

The first step in the design process is to elicit user requirements. This is done by interviewing a set of potential users. A key requirement is that all types of possible users be interviewed. They should also represent the proper experience level of the actual users.

\subsection{Identification of Experts}

The first step in determining user requirements is to identify experts that represent all possible user types for the VC system. This requires some knowledge of the domain and also of the goals of the VC.

Potential users of the VC device include astronauts, mission operations personnel and scientists.

\subsection{Usability Survey}

A survey of potential users and stakeholders for the VC system has been developed. The main topics for the survey include background, general system uses, interface type and data display parameter formats.

Brief scenarios and interaction diagrams have also been developed. These are presented to users to show the basic concept of the VC and to get feedback of what would be a beneficial interface for a particular user and what may also not be of benefit to a user. Scenarios and use cases give users examples of how the VC can be applied.

\subsection{Use Cases}

Use cases allow possible users to see the usefulness of the $\mathrm{VC}$ as well as suggest improvements or changes to make the $\mathrm{VC}$ a more efficient system. The following is an example use case where mission planners interact with the VC to plan a future traverse.

\subsubsection{Use Case for the VC for Planning a Surface Traverse: Identify Next Days Traverse}

Summary: Mission Planners Review Virtual Camera (VC) data to determine next day's traverses across the planetary surface Basic Course of Events: 
1. Science and surface data collected into a database from previous missions and previous traverses correlating various missions together 2. Planners use a haptic input system which provided force feedback for terrain with the ability to correlate feedback with various mission parameters: minerals, resources, slope, and obstacles.

3. Planners gather around a large live board allowing group interaction to determine both a safe and exploration fruitful day's worth of activities

4. The system records everything the user does annotating areas of interest by judging how long the planner stays observing a particular area or region

5.Group Elicitation Method style interface to allow traverse decision making in a group

6. A final traverse map is uplinked to rover system

Possible Failures:

Database not of high enough resolution to make a proper determination

Possible solution:

Have rover collect more data of area before moving

Extension:

1. Traverse direction determined by:

Science return

Resource requirements for surface base

Safety

2. Traverse also selected to assist other nearby rovers

Preconditions:

Mission defined rules for safety and mission success criteria

Post-conditions:

Surface exploration following mission rules

\section{Prototype Development}

\subsection{Development Plan}

Once user requirements have been defined the VC system needs to be prototyped. This allows humanin-the-loop simulations to take place where the system can be tested with a variety of scenarios.

Users can interact with these actual system prototypes which are developed on the actual tablet-based computer system that will be used for the end product.

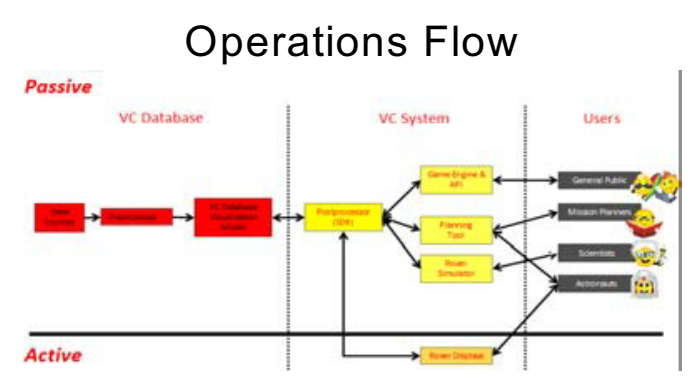

Figure 3 Operations Flow for the Virtual Camera for Planetary Exploration

This then allows the full functionality to be incorporated after initial user testing and feedback.

Figure 3 shows the operations flow for the $\mathrm{VC}$, the database and end users. The VC system interfaces, or interacts with the VC database of terrain data, science data and mission data. Other information could include consumables available and radiation or other dangers. Users then use the tablet-based computer system to interact using advanced interface techniques.

\subsection{Software Development Tools}

The VC development plan is to be completed in stages. The initial stage is an implementation of the $\mathrm{VC}$ on a portable handheld tablet-style computing device. This allows advanced action media concepts to be tested including finger-based controls and screen movement based on moving the entire device. The usefulness of these new human-machine interaction capabilities in the space environment will be analyzed.

The software development effort is using the Android software development kit (SDK) and unity 3-D development system for data display. Integrated with a terrain model of the desired exploration site, such as an asteroid, will be other simulated science data.

\subsection{Horizontal Prototypes}

Basic interface screens will be developed and will soon be ready for user testing. These screens show all of the functionality and capabilities of the VC although the database interactivity will not yet be possible. This horizontal prototyping allows astronaut, scientist and mission operations personnel to provide feedback as to the usefulness of the screens being developed. Emergent uses of the system and behaviors enabled or limited by the VC will be discovered. Figure 4 shows an example horizontal prototype with areas of interest, areas of safety concern and multi-agent external views. 


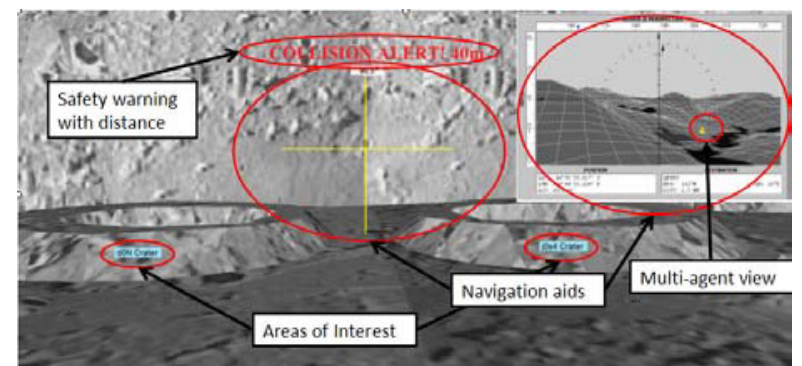

Figure 4 Prototype Screen for VC showing Areas of Interest and of Safety Concern with Possible use of Colors and Symbols

We can use screens such as Figure 4 to help further define requirements for:

- Safety resolution requirements

- Exploration resolution requirements

- Realtime data display requirements

- Format of data displays (visualization)

- Graphical User Interfaces

Types of interfaces (visual and auditory) will be examined to determine their usefulness in the VC system. For instance, for astronauts the visual sense is oftentimes already overloaded. It may be useful then to use auditory information and warning messages. Voice command could also be useful to interface with the VC for them.

An important type of test will be system memorability since key users will operate as casual users (astronauts) and will not have easy access to refresher training on another remote surface.

\subsection{Vertical Prototypes}

For a subset of the overall VC design a vertical prototype will be developed. This will allow complete testing of interactivity of the system. It will also help to answer questions such as:

- How can we minimize computationally intensive data requirements (triangulation, referen tial data, go-no go instead of continuous values, etc)?

- How can learning be implemented into design? Other capabilities to be tested include the ability to integrate real-time sensor data into the VC database with the learning feature. It is also desired to capture expert knowledge into database and represent that knowledge in a human-machine interface (HMI).

\section{Conclusion}

The Virtual Camera for planetary exploration will assist in future exploration involving humans and robots. It is designed to provide improved situational awareness and augmented reality to define areas of interest and safety concern on remote planetary bodies. It will act as remote agents for mission planners and scientists capturing their knowledge and expertise as astronauts explore the solar system. Humancentered-design techniques are being applied to the development of this tool involving all potential user groups from the beginning of the design process.

The design process begins with defining potential users and how they may take advantage of the VC. These users are interviewed and the resulting feedback incorporated into display format prototypes that can be tested by users. This iterative process results in a final product that has already been tested by users and is designed with their needs in mind. This includes the multi-agent uses and requirements of the VC.

Data fusion is a critical design element of the VC and a preliminary analysis of data requirements and fusion considerations was also presented.

\section{References}

[1] Boy, Guy, et al., "The Virtual Camera: A Third Person View," Third International Conference on Applied Human Factors and Ergonomics, July 2010.

[2] Huadong Wu, "Sensor Data Fusion for Context-Aware Computing Using Dempster-Shafer Theory," Ph.D. dissertation, Carnegie Mellon University, December 2003.

[3] Deans, Matthew., et al, "Field Testing Next-Generation Ground Data Systems for Future Missions", 42nd Lunar and Planetary Science Conference, 2011.

[4] Estlin, Tara., et al, "Increased Mars rover autonomy using AI planning, scheduling and execution.", Proceedings of the IEEE International Conference on Robotics and Automation, Rome, Italy, 2007. 